Research Article

\title{
Quartic Integral in Rigid Body-Gyrostat Dynamics
}

\author{
C. Mnasri $(\mathbb{D})$ and A. A. Elmandouh $\mathbb{D}^{1,2}$ \\ ${ }^{1}$ Department of Mathematics and Statistics, College of Science, King Faisal University, P.O. Box 400, \\ Al-Ahsa 31982, Saudi Arabia \\ ${ }^{2}$ Department of Mathematics, Faculty of Science, Mansoura University, Mansoura 35516, Egypt
}

Correspondence should be addressed to A. A. Elmandouh; aelmandouh@kfu.edu.sa

Received 8 October 2020; Revised 7 November 2020; Accepted 18 November 2020; Published 11 December 2020

Academic Editor: Elbaz Abouelmagd

Copyright (C) 2020 C. Mnasri and A. A. Elmandouh. This is an open access article distributed under the Creative Commons Attribution License, which permits unrestricted use, distribution, and reproduction in any medium, provided the original work is properly cited.

\begin{abstract}
In this work, we investigate the problem of constructing new integrable problems in the dynamics of the rigid body rotating about its fixed point as results of the effect of a combination of potential and gyroscopic forces possessing a common symmetry axis. We introduce two new integrable problems in a rigid body dynamics that generalize some integrable problems in this field, known by names of Chaplygin and Yehia-Elmandouh.
\end{abstract}

\section{Introduction}

One of the classical problems manifesting in mathematical physics is the issue of determining whether a dynamical system, especially a mechanical one, is integrable or not. Integrability in this context often points out to Liouville integrability. The Liouville integrability concept is defined as the Hamiltonian system with $n$ degrees of freedom that is completely integrable if it has $n$ independent integrals of motion which are in involution, i.e., their Poisson brackets are zero [1]. The integrable systems possess miscellaneous properties such as their behavior that can be globally tested in an infinite interval of time, gratitude to the theories of perturbation, those systems that can be applied to give an appointed inference about the nonintegrable systems nearby them, and in general, the motion equations can be solved by quadratures [2]. The problem of integrability is split into two categories. The first one is finding the sufficient conditions for the integrability, and this requires the construction of a sufficient number of first integrals of motion. Numerous methods can be utilized to construct the first integrals of motion such as the direct method, Darboux method, and Yehia method (see, e.g., [3-13]). The second one deals with obtaining the necessary conditions of the integrability (see, e.g., [14-20]), but we must introduce the required number of the integrals to confirm the integrability.

One of the significant issues in applications in assorted branches of science such as physics and astronomy is the problem of a rigid body and its extension to a gyrostat (see, e.g., [21-23]). So, it is a beneficial model for research from different points of view [24-29]. Consequently, the present work interested in analyzing the general motion of a rigid body about its fixed point that happens under the effect of a combination of potential (velocity-independent) forces and gyroscopic (velocity-dependent) forces. The gyroscopic forces are specified by $l=\left(0,0, l_{3}\right)$, while the potential forces are characterized by $V(\gamma)$. As it outlined in [30], this motion can be characterized by the Lagrangian:

$$
L=\frac{1}{2} \omega \cdot \omega I+l \cdot \omega-V,
$$

where $\omega=(p, q, r)$ is the angular velocity, and $I=\operatorname{diag}(A, B, C)$ is the inertia matrix of the body. The equations of motion corresponding Lagrangian (1) are [30-32]

$$
\dot{\omega} I+\omega \times(\omega I+\mu)=\gamma \times \frac{\partial V}{\partial \gamma}, \quad \dot{\gamma}+\omega \times \gamma=0,
$$


where $\gamma=\left(\gamma_{1}, \gamma_{2}, \gamma_{3}\right)$ is the unit vector which is fixed upward in space, and $\mu$ is expressed as

$$
\mu\left(\gamma_{1}, \gamma_{2}, \gamma_{3}\right)=\frac{\partial}{\partial \gamma}(l \cdot \gamma)-\left(\frac{\partial}{\partial \gamma} \cdot 1\right) \gamma .
$$

It is well-known that the Euler-Poisson equation (2) has three classical integrals of motion. They are as follows:

(1) Area integral:

$$
I_{1}=(\omega \mathrm{I}+1) \cdot \gamma=f
$$

where the arbitrary constant $f$ denotes the value of area integral. This integral is sometimes named as a cyclic integral due to it correspondences the cyclic variable $\psi$, the angle of precession.

(2) Jacobi integral:

$$
I_{2}=\frac{1}{2} \omega \cdot \omega I+V=h,
$$

where the arbitrary constant $h$ identifies the value of the Jacobi integral.

(3) Geometric integral:

$$
I_{3}=\gamma \cdot \gamma=1
$$

Taking into account the Jacobi theorem on the last integrating multiplier [33], four integrals of motion are needed to confirm the integrability of the equation of motion (2). Thence, the existence of a fourth integral independent of those (4), (5), and (6) is sufficient to prove the integrability. It is worth noticing that the integrable case either generally integrable or conditionally integrable according to the fourth integral is either valid on an arbitrary level of the cyclic integral $I_{1}$ or valid on a single level of cyclic integral $I_{1}$ which is usually zero.

The problem of a rigid body which is described by the equation of motion (2) was studied in diverse posterior works from the point of view of the integrability. Those works include three types of problems.

The first problem deals with the problem of the motion of a rigid body about a fixed point under the action of its weight. It is characterized by $V=\mathrm{r}_{0} \cdot \gamma$, and $\mu=0$, where $\mathrm{r}_{0}$ is a constant vector that represents the center of the mass vector. It attracted the attention of the researchers for a long time, nearly two and a half centuries, and thus, it has a great history. It includes three (no more) general integrable problems bearing the names of who discovered them, Euler, Lagrange, and Kowalevski, and one conditional integrable problem of Goriatchev-Chaplygin (see, e.g., [34]).

The second problem concerns the motion of a rigid body about its fixed point under the effect of its weight, and moreover, there is a rotor spinning about its axis of symmetry which is fixed in the body with a constant angular velocity. It is worth mentioning that it is a simple multibody that consists of the main body and the rotor, and it is termed in literatures a gyrostat. The second problem regards as a generalization to the first problem, and it is determined by $V=\mathrm{r}_{0} \cdot \gamma$ and $\mu=k$, where $k$ is a constant vector characterizing the gyroscopic moment due to the existence of the rotor. It contains three general and one conditional integrable problems generalizing those in the first problem by adding the gyrostatic moment. The general cases are Lagrange, Joukovsky [34], and Yehia [35], while the conditional case is the Sretensky case which generalizes the Goriatchev-Chaplygin case in the first problem. In [36], the author proved that the equations of the motion for the current problem does not own more than the three mentioned cases.

The third problem studies the problem of the motion of a rigid body in an incompressible ideal fluid, infinitely extending and at rest at infinity. The simple connected body is either described by the traditional Kirchoff equations [37] or by their Hamiltonian [38] form, while the body bounded by a multiconnected surface is described either by Lamb equations [39] or by its equivalent Hamiltonian form (see, e.g., [34]). The utilization of the equations of Kirchhoff and Lamb to describe this problem lacks to demonstrate the link between this problem and the other problems of rigid body dynamics. The link between both problems is proved by Yehia who introduced the equations of motion for a rigid body in a liquid by removing the translation motion that appears as cyclic variables (see, [40]), and the reduced problem is described by $V=r_{0} \cdot \gamma+(1 / 2) \gamma \mathrm{J} \cdot \gamma$ and $\mu=k-2 \gamma \overline{\mathrm{K}}$, where $J$ and $\bar{K}$ are the constant $3 \times 3$ matrices. The integrable cases for the third problem have been introduced in $[34,40]$.

To dodge the ambiguity, we summarize those problems in Table 1. Obviously, each problem is a generalization of the previous one by inserting some of the additional parameters, which represented terms having certain physical interpretations.

According to the methodology used, this work deals only with two-dimensional mechanical systems, as it is outlined down in section two. It is obvious that this problem has three degrees of freedom in which one of them can be ignored due to the presence of a cyclic variable, precession angle, by utilizing the Routh procedure. Thence, the current problem can be characterized by Routhian (see, [41]).

$$
\begin{aligned}
R= & \frac{1}{2}\left[\frac{\dot{\gamma}^{2}}{1-\gamma_{3}^{2}}+\frac{C\left(1-\gamma_{3}^{2}\right) \dot{\varphi}^{2}}{A-(A-C) \gamma_{3}^{2}}\right]+\frac{f\left(C \gamma_{3}+A l_{3}\left(1-\gamma_{3}^{2}\right)\right)}{A\left[A-(A-C) \gamma_{3}^{2}\right]} \dot{\varphi} \\
& -\frac{1}{A}\left(V+\frac{\left(f-l_{3} \gamma_{3}\right)^{2}}{2\left[A-(A-C) \gamma_{3}^{2}\right]}\right) .
\end{aligned}
$$

One can do the transformation,

$$
\mathrm{d} t=\frac{C\left(1-\gamma_{3}^{2}\right)}{A-(A-C) \gamma_{3}^{2}} \mathrm{~d} \tau
$$


TABLE 1: Different problems in a rigid body dynamics.

\begin{tabular}{lccc}
\hline No. & Problem & Potential function $V$ & $\begin{array}{c}\text { Vector } \\
\text { function } \mu\end{array}$ \\
\hline 1 & Heavy rigid body & $V=r_{0} \cdot \gamma$ & $\mu=\mathbf{0}$ \\
2 & Heavy gyrostat & $V=\mathbf{r}_{0} \cdot \gamma$ & $\mu=\mathbf{k}$ \\
3 & Rigid body in a liquid & $V=\mathbf{r}_{0} \cdot \gamma+(1 / 2) \gamma \mathbf{J} \cdot \gamma$ & $\mu=\mathbf{k}-2 \gamma \overline{\mathbf{K}}$ \\
\hline
\end{tabular}

to Routhian (7), and we get

$$
\begin{aligned}
R_{0}= & \frac{A}{2}\left[\dot{\varphi}^{2}+\frac{A-(A-C) \gamma_{3}^{2}}{C\left(1-\gamma_{3}^{2}\right)^{2}} \hat{\gamma}_{3}^{2}\right]+\frac{f C \gamma_{3}+A l_{3}\left(1-\gamma_{3}^{2}\right)}{\left[A-(A-C) \gamma_{3}^{2}\right]} \dot{\varphi} \\
& -\frac{C\left(1-\gamma_{3}^{2}\right)}{\left[A-(A-C) \gamma_{3}^{2}\right]}\left[V+\frac{\left(f-l_{3} \gamma_{3}\right)^{2}}{2\left[A-(A-C) \gamma_{3}^{2}\right]}\right]
\end{aligned}
$$

where $\tau$ is the fictitious time, and dash refers to the derivative with respect to $\tau$.

\section{Basic Equations}

A method for constructing the two-dimensional integrable mechanical systems in which the additional integral is a polynomial in velocities has been presented by Yehia in [42], and it has been developed in [43]. This method has been successfully applied to construct new integrable problems (not necessarily plane) whose complementary integral is a polynomial in velocities up to degree four (e.g., [4-6, 44-49]). This method is restrictively employed for two mechanical systems. There are a wide class of beforementioned systems such as the $n$-dimensional mechanical systems admitting $(n-2)$ cyclic variables and the particle motion on a smooth surface under the influence of distinct types of forces. A further example is a present problem which describes the rotation of a rigid body about a fixed point under the effect of potential and gyroscopic forces possessing a common axis of symmetry, so the motion has a cyclic variable, and this enables us to apply Routh procedure to lessen the degrees of freedom from three to two $[32,33]$.

The two-dimensional mechanical systems are described by Lagrangian equation.

$$
L=\frac{1}{2}\left(b_{11} \dot{q}_{1}^{2}+2 b_{12} \dot{q}_{1} \dot{q}_{2}+b_{22} \dot{q}_{2}^{2}\right)+b_{1} \dot{q}_{1}+b_{2} \dot{q}_{2}-V,
$$

where the functions $b_{i j}, b_{i}$, and $V$ rely on the generalized coordinates $q_{1}$ and $q_{2}$, and dots refer to the derivatives with respect to the time $t$. Birkhoff theorem [50] guarantees the existence of a certain canonical transformation which is applied to turn Lagrangian (10) into

$$
L=\frac{\Lambda}{2}\left(\dot{\xi}^{2}+\dot{\eta}^{2}\right)+l_{1} \dot{\xi}+l_{2} \dot{\eta}-V
$$

where $\Lambda, l_{1}, l_{2}$, and $V$ are the functions in the two variables $x$ and $y$. The usefulness of this step is to diminish the number of functions from six to four. It is obvious that the Lagrangian (10) has a Jacobi integral in the form

$$
I_{1}=\frac{\Lambda}{2}\left(\dot{\xi}^{2}+\dot{\eta}^{2}\right)+V=h,
$$

where $h$ is an arbitrary constant. According to Liouville theorem for the equivalent Hamiltonian system, system (11) is completely integrable if it has another first integral independent on the Jacobi integral (12).

Executing the time transformation (see Appendix A for more details about time transformation),

$$
\mathrm{d} t=\Lambda \mathrm{d} \tau
$$

to Lagrangian (11), we get

$$
L_{0}=\frac{1}{2}\left(\xi^{\prime^{2}}+\eta^{\prime^{2}}\right)+l_{1} \xi^{\prime}+l_{2} \eta^{\prime}-U
$$

where $U=\Lambda(h-V)$, and I refers to the derivative with respect to $\tau$. The Lagrangian equations corresponding to Lagrangian (14) are

$$
\begin{aligned}
& \xi^{\prime \prime}+\Omega \eta^{\prime}=\frac{\partial U}{\partial \xi} \\
& \eta^{\prime \prime}+\Omega \xi^{\prime}=\frac{\partial U}{\partial \eta}
\end{aligned}
$$

where $\Omega=\partial l_{1} / \partial \eta-\partial l_{2} / \partial \xi$. This system has a Jacobi integral in the form

$$
I_{1}=\frac{1}{2}\left(\xi^{\prime^{2}}+\eta^{\prime^{2}}\right)+U=0
$$

Now, we are going to find an additional first integral that is independent on the Jacobi integral (16). Based on [42], the complementary integral that is assumed to be quartic in velocities can be expressed as

$$
I_{2}=\xi^{\prime^{4}}+P_{3} \xi^{\prime^{3}}+Q_{3} \xi^{\prime^{2}} \eta^{\prime}+P_{2} \xi^{\prime^{2}}+Q_{2} \xi^{\prime} \eta^{\prime}+P_{1} \xi^{\prime}+Q_{1} \eta^{\prime}+R,
$$

where the functions $P_{j}, Q_{j}$, and $R$ depend on the two variables $\xi$ and $\eta$. Calculating the derivative of (17) with respect to $\tau$ and using the Jacobi integral (16) to remove all the even powers of $\eta^{\prime}$ as in [42], we get the following nonlinear system of partial differential equations:

$$
M . D_{\xi} X+N . D_{\eta} X=B \text {, }
$$

where $X=\left(\begin{array}{lllllllll}P_{1} & P_{2} & P_{3} & Q_{1} & Q_{2} & Q_{3} & R & U & \Omega\end{array}\right)^{T}$ is the vector of the unknown functions. $D_{\xi} X$ and $D_{\eta} X$ are partial derivatives according to the variables $\xi$ and $\eta$ of the vector $X . M$ and $N$ are the matrices and given as follows: 


$$
M=\left(\begin{array}{ccccccccc}
1 & 0 & 0 & 0 & 0 & 0 & 0 & 3 P_{3} & 0 \\
1 & 0 & 0 & 0 & 0 & 0 & 0 & 2 Q_{3} & 0 \\
0 & 1 & 0 & 0 & 0 & 0 & 0 & 0 & 0 \\
0 & 1 & 0 & 0 & 0 & 0 & 0 & 0 & 0 \\
0 & 0 & 1 & 0 & 0 & 0 & 0 & 0 & 0 \\
0 & 0 & 1 & 0 & 0 & 0 & 0 & 0 & 0 \\
1 & 0 & 0 & 0 & 0 & 0 & 1 & 2 P_{1} & 0 \\
1 & 0 & 0 & 0 & 0 & 0 & 1 & P_{1} & 0 \\
1 & 0 & 0 & 0 & 0 & 0 & 1 & Q_{2} & 0
\end{array}\right),
$$

$$
N=\left(\begin{array}{ccccccccc}
0 & 0 & 0 & -1 & 0 & 2 U & 0 & Q_{3} & 0 \\
0 & 0 & 0 & 1 & 0 & 0 & 0 & 0 & 0 \\
0 & 0 & 0 & 0 & -1 & 0 & 0 & 0 & 0 \\
0 & 0 & 0 & 0 & 1 & 0 & 0 & 0 & 0 \\
0 & 0 & 0 & 0 & 0 & -1 & 0 & 0 & 0 \\
0 & 0 & 0 & 0 & 0 & 1 & 0 & 0 & 0 \\
0 & 0 & 0 & 0 & 2 U & 0 & 1 & Q_{2} & 0 \\
0 & 0 & 0 & 2 U & 0 & 0 & 1 & Q_{1} & 0 \\
0 & 0 & 0 & 0 & 0 & 0 & 1 & 0 & 0
\end{array}\right) .
$$

The right-side vector $B$ is given by $B=$

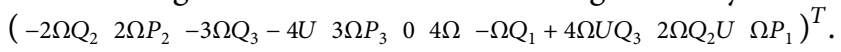

System (18) composed of nine nonlinear partial differential equations with nine unknown functions is not easy to solve exactly. Notice that the solution of this system determines a two-dimensional integrable system with an additional quartic integral in the velocities which is valid on a zero level of Jacobi integral.

The sixth equation in (18), which is $\partial P_{3} / \partial \xi_{+}$ $\partial Q_{3} / \partial \eta=4 \Omega$, and the definition of $\Omega$, allow us to write Lagrangian (14) in the form

$$
L_{0}=\frac{1}{2}\left(\xi^{\prime^{2}}+\eta^{\prime^{2}}\right)+\frac{1}{4}\left(P_{3} \xi^{\prime}-Q_{3} \eta^{\prime}\right)-U
$$

\section{Applications to Rigid Body Dynamics}

In the present section, we investigate the construction of new integrable systems with a quartic integral in the dynamics of a rigid body movement. Seeing that the metric corresponding to Lagrangian (9) is $\mathrm{d} s^{2}=\mathrm{d} \varphi^{2}+g\left(\gamma_{3}\right) \mathrm{d} \gamma_{3}^{2}$, it is more suitable to use the variable $p$ instead of $\eta$ through the relation

$$
\eta=\int \frac{\mathrm{d} p}{f(p)},
$$

where

$$
f(p)=\sqrt{\left(p-p_{1}\right)\left(p-p_{2}\right)\left(p-p_{3}\right)\left(p+p_{1}+p_{2}+p_{3}\right)},
$$

where $p_{1}, p_{2}$, and $p_{3}$ are the arbitrary parameters. We consider a certain class of problems in a rigid body dynamics in which the gyroscopic forces are determined by

$$
\Omega(\xi, p)=a_{1} \Omega_{1}(p) \cos \xi+a_{2} \Omega_{0}(p),
$$

and the potential forces are characterized by

$$
\begin{aligned}
U(\xi, p)= & u(p)+a_{3} v(p) \sin \xi-\frac{a_{1} a_{2}}{8} f_{0}(p) \cos \xi \\
& +a_{1}^{2} m(p) \cos 2 \xi,
\end{aligned}
$$

where $a_{1}, a_{2}$, and $a_{3}$ are the arbitrary constants. The motivation for the choice of the two functions (24) and (25) is that they represent a large class of problems in the dynamics of a rigid body. Certain clarifications should be made for two particular cases.

(1) Time-reversible case: this case is characterized by the absence of gyroscopic forces, i.e., $\Omega=0$. This happens if $a_{1}=0$ and $a_{2}=0$, The potential function (25) reduces to $U=u(p)+a_{3} v(p) \sin \xi$ which is a Kowalevski-type potential. Furthermore, if we change $\xi \longrightarrow 2 \xi$, the potential function takes the form $U=u(p)+a_{3} v(p) \sin 2 \xi$ which is a Chaplygin-type potential. The previous studies concerning those types of potentials lead to several generalizations for integrable problems in the rigid body dynamics in which the additional integral is a quartic polynomial in the velocities (e.g., [51-53]). This type of problem is referred in literatures as time-reversible systems.

(2) Time-irreversible case: this case involves a gyroscopic forces acting on the motion besides the potential forces. We can split it into two subcases.

(a) When setting $a_{1}=0$, the gyroscopic forces is characterized by $\Omega=a_{2} \Omega_{0}(p)$, and the potential function (25) becomes $U=u(p)+a_{3} v(p) \sin \xi$. The potential function is a type of Kowalevskigyrostat type potential or Chaplygin-gyrostat type potential (if $\xi \longrightarrow 2 \xi$ ). This problem has been studied in several works such as $[51,52]$. These studies lead to a generalization of a Kowalevski case and Chaplygin case by adding a constant gyrostatic moment.

(b) When $a_{1} a_{2} \neq 0$, the full structure of $\Omega$ and $U$ is considered in [49], but the authors solved the basic equations for a special cases leading to the Kowalevski case, and they introduced two integrable problems generalize Kowalevski case and Sokolov case.

Inserting the expressions (22), (24), and (25) into the equations (18)-(21), we have 


$$
\begin{aligned}
L= & \frac{1}{2}\left(\eta^{\prime^{2}}+\frac{p^{\prime^{2}}}{f(p)}\right)+\left(a_{1} J_{1}(p) \cos \xi+a_{2} J_{0}(p)\right) \xi^{\prime}+u(p) \\
& +a_{3} v(p) \sin \xi-\frac{a_{1} a_{2}}{8} f_{0}(p) v(p) \cos \xi+a_{1}^{2} m(p) \cos (2 \xi)
\end{aligned}
$$

$$
\begin{aligned}
I= & \xi^{{ }^{4}}+P_{3} \xi^{3}+\bar{Q}_{3} \xi^{\prime^{2}} p^{\prime}+P_{2} \xi^{\prime^{2}}+\bar{Q}_{2} \xi^{\prime} p^{\prime}+P_{1} \xi^{\prime}+\bar{Q}_{1} p^{\prime} \\
& +R=\varepsilon_{0},
\end{aligned}
$$

where $\bar{Q}_{j}=\left(Q_{j} / \sqrt{f(p)}\right)$. In what follows, we inscribe $Q$ instead of $\bar{Q}$ for simplicity. Taking into account the transformation (22) and inserting the two expressions (24) and (25) into the basic equation (18), we get the following nonlinear system of partial differential equations in the following form:

$$
\begin{aligned}
& Q_{3} \frac{\mathrm{d} f}{\mathrm{~d} p}+2 f \frac{\partial Q_{3}}{\partial p}-2 \frac{\partial P_{3}}{\partial \xi}=0, \\
\left(8 a_{3} v+\right. & \frac{\partial P_{3}}{\partial p}+\frac{\partial Q_{3}}{\partial \xi}-4 a_{2} \Omega_{0}-4 a_{1} \Omega_{1} \cos \xi=0,
\end{aligned}
$$




$$
\begin{aligned}
\frac{\partial R}{\partial p}= & 2 a_{1}^{2} m Q_{2} \sin 2 \xi-\frac{a_{1} a_{2}}{8} Q_{2} f_{0} v \sin \xi+a_{1} P_{1} \Omega_{1} \cos \xi-\frac{a_{1} a_{2}}{8} Q_{2} f_{0} v \sin 3 \xi+a_{2} P_{1} \Omega_{0} \\
& a_{1}^{2}\left\{16 f m \frac{\partial Q_{1}}{\partial p}+a_{2} f f_{0} v Q_{2} \Omega_{1}-16 a_{2} f m Q_{2} \Omega_{0}+8 Q_{1} m \frac{\mathrm{d} f}{\mathrm{~d} p}+8 Q_{1} f \frac{\mathrm{d} m}{\mathrm{~d} p}\right\} \cos 2 \xi-8 a_{1}^{3} f Q_{2} m \Omega_{1} \cos 3 \xi \\
& +\left\{-a_{1} a_{2} f_{0} v Q_{1} \frac{\mathrm{d} f}{\mathrm{~d} p}+2 a_{1} a_{2}^{2} f f_{0} Q_{2} \Omega_{0}-a_{1} a_{2} Q_{1} f f_{0} \frac{\mathrm{d} v}{\mathrm{~d} p}-16 a_{1} f u Q_{2} \Omega_{1}-8 a_{1}^{3} f m Q_{2} \Omega_{1}-a_{1} a_{2} v f Q_{1} \frac{\mathrm{d} f_{0}}{\mathrm{~d} p}\right. \\
& \left.+8 a_{1}^{3} v P_{1}-2 a_{1} a_{2} f_{0} f v \frac{\partial Q_{1}}{\partial p}\right\} \cos \xi+\left\{16 a_{3} f v \frac{\partial Q_{1}}{\partial p}+8 a_{3} v Q_{1} \frac{\mathrm{d} f}{\mathrm{~d} p}-16 a_{2} a_{3} f Q_{2} v \Omega_{0}+a_{1} a_{2} P_{1} f_{0} v+8 a_{3} f Q_{1} \frac{\mathrm{d} v}{\mathrm{~d} p}\right\} \sin \xi \\
& -8 a_{1}\left(2 a_{1} m P_{1}+a_{3} v f Q_{2} \Omega_{1}\right) \sin 2 \xi+8 Q_{1} u \frac{\mathrm{d} f}{\mathrm{~d} p}+a_{1}^{2} a_{2} f Q_{2} f_{0} v-16 a_{2} f Q_{2} u \Omega_{0}+16 f u \frac{\partial Q_{1}}{\partial p}+8 Q_{1} f \frac{\mathrm{d} u}{\mathrm{~d} p}=0,
\end{aligned}
$$

where

$$
\begin{aligned}
& \Omega_{0}=\frac{\mathrm{d} J_{0}}{\mathrm{~d} p}, \\
& \Omega_{1}=\frac{\mathrm{d} J_{1}}{\mathrm{~d} p} .
\end{aligned}
$$

It is obvious that systems (28)-(35) are a nonlinear system of partial differential equations, and so in general, their solution is somewhat difficult. For simplicity, we turn these equations to the system of ordinary differential equations by setting the integral coefficients in a more suitable form. After some trials, the integral coefficients can be expressed as follows:

$$
\begin{aligned}
& P_{3}(\xi, p)=a_{1} f_{0}(p)+a_{1} f_{1}(p) \cos \xi \\
& Q_{3}(\xi, p)=8 a_{1} \sin \xi \\
& P_{2}(\xi, p)=P_{0}(p)+16 a_{3} p \sin \xi+a_{1} a_{2} G(p) \cos \xi+a_{1}^{2} T(p) \cos 2 \xi \\
& Q_{2}(\xi, p)=a_{3} q_{1}(p) \cos \xi+a_{1} a_{2} q_{2}(p) \sin \xi+a_{1}^{2} q_{3}(p) \sin 2 \xi \\
& P_{1}(\xi, p)=f_{3}(p) \cos \xi+a_{1}^{2} a_{2} f_{4}(p) \cos 2 \xi+a_{1}^{3} f_{5}(p) \cos 3 \xi+a_{1} a_{3} f_{6}(p) \sin 2 \xi+a_{2} a_{3} f_{7}(p) \sin \xi+f_{8}(p) \\
& Q_{1}(\xi, p)=a_{2} a_{3} f_{9}(p) \cos \xi+a_{1} a_{3} f_{10}(p)(\cos 2 \xi+1)+a_{1} f_{11}(p) \sin \xi+a_{2} a_{1}^{2} f_{12}(p) \sin 2 \xi+a_{1}^{3} f_{13}(p) \sin 3 \xi
\end{aligned}
$$

Inserting the expressions of the integral's coefficients (37) into the equations (28)-(35) and equating the coefficients of trigonometric functions to zero, we obtain the following system of ordinary differential equations:

$$
\begin{aligned}
a_{1}\left(f_{1}+4 \frac{\mathrm{d} f}{\mathrm{~d} p}\right) & =0 \\
a_{2} f\left(\frac{\mathrm{d} f_{0}}{\mathrm{~d} p}-4 \Omega_{0}\right) & =0 \\
a_{1}\left(4 \Omega_{1}-\frac{\mathrm{d} f_{1}}{\mathrm{~d} p}-8\right) & =0 \\
a_{3}\left(q_{1} \frac{\mathrm{d} f}{\mathrm{~d} p}+2 f \frac{\mathrm{d} q_{1}}{\mathrm{~d} p}-32 p-8 v\right) & =0
\end{aligned}
$$




$$
\begin{aligned}
& a_{1} a_{2}\left(q_{2} \frac{\mathrm{d} f}{\mathrm{~d} p}+2 f \frac{\mathrm{d} q_{2}}{\mathrm{~d} p}+2 G-f_{0} v-48 f \Omega_{0}\right)=0, \\
& a_{1}^{2}\left(q_{3} \frac{\mathrm{d} f}{\mathrm{~d} p}+2 f \frac{\mathrm{d} q_{3}}{\mathrm{~d} p}+4 T+16 m-24 f \Omega_{1}\right)=0, \\
& a_{1} a_{2}\left(\frac{\mathrm{d} G}{\mathrm{~d} p}-3 f_{0} \Omega_{1}-3 f_{1} \Omega_{0}-q_{2}\right)=0, \\
& a_{1}^{2}\left(2 \frac{\mathrm{d} T}{\mathrm{~d} p}-3 f_{1} \Omega_{1}+4 q_{3}\right)=0 \\
& a_{3}\left(q_{1}-16\right)=0 \text {, } \\
& \frac{\mathrm{d} P_{0}}{\mathrm{~d} p}-3 a_{2}^{2} f_{0} \Omega_{0}-\frac{3}{2} a_{1}^{2} f_{1} \Omega_{1}=0, \\
& a_{2} a_{3}\left(f_{9} \frac{\mathrm{d} f}{\mathrm{~d} p}+2 f \frac{\mathrm{d} f_{9}}{\mathrm{~d} p}-2 f_{7}-6 f_{0} v-4 f q_{1} \Omega_{0}\right)=0 \\
& a_{1} a_{3}\left(f_{10} \frac{\mathrm{d} f}{\mathrm{~d} p}+2 f \frac{\mathrm{d} f_{10}}{\mathrm{~d} p}+8 \frac{\mathrm{d}}{\mathrm{d} p}(v p)-4 f_{6}-3 f_{1} v-2 q_{1} f \Omega_{1}\right)=0, \\
& f_{3}+a_{1}\left(f \frac{\mathrm{d} f_{11}}{\mathrm{~d} p}+\frac{1}{2} f_{11} \frac{\mathrm{d} f}{\mathrm{~d} p}-8 f \frac{\mathrm{d} u}{\mathrm{~d} p}-8 u \frac{\mathrm{d} f}{\mathrm{~d} p}\right)+a_{1}^{3}\left(4 m \frac{\mathrm{d} f}{\mathrm{~d} p}+3 m f_{1}\right)+a_{1} a_{2}^{2}\left(\frac{3}{8} f_{0} v-2 f q_{2} \Omega_{0}\right)=0, \\
& a_{2} a_{1}^{2}\left(8 f_{12} \frac{\mathrm{d} f}{\mathrm{~d} p}+16 f \frac{\mathrm{d} f_{12}}{\mathrm{~d} p}+8 \frac{\mathrm{d}}{\mathrm{d} p}\left(f_{0} v f\right)+32 f_{4}+96 f_{0} m-32 f q_{3} \Omega_{0}-32 f q_{3} \Omega_{0}-16 q_{2} f \Omega_{1}\right)=0, \\
& a_{1}^{3}\left(2 f \frac{\mathrm{d} f_{13}}{\mathrm{~d} p}+f_{13} \frac{\mathrm{d} f}{\mathrm{~d} p}+6 f_{5}-8 \frac{\mathrm{d}}{\mathrm{d} p}(m f)-2 q_{3} f \Omega_{1}+6 m f_{1}\right)=0, \\
& a_{1} a_{3}\left(f_{10} \frac{\mathrm{d} f}{\mathrm{~d} p}+2 f \frac{\mathrm{d} f_{10}}{\mathrm{~d} p}-8 \frac{\mathrm{d}}{\mathrm{d} p}(v f)-2 q_{1} f \Omega_{1}-3 f_{1} v\right)=0, \\
& a_{1}\left(2 P_{0} \Omega_{1}-f_{11}\right)+2 a_{1} a_{2}^{2} G \Omega_{0}+a_{1}^{3}\left(16 m+T \Omega_{1}\right)-\frac{\mathrm{d} f_{3}}{\mathrm{~d} p}=0, \\
& a_{1}^{2} a_{2}\left(2 T \Omega_{0}-2 f_{12}+f_{0} v+G \Omega_{1}-\frac{\mathrm{d} f_{4}}{\mathrm{~d} p}\right)=0 \\
& a_{1}^{3}\left(T \Omega_{1}-3 f_{13}-16 m-\frac{\mathrm{d} f_{5}}{\mathrm{~d} p}\right)=0 \\
& a_{2} a_{3}\left(\frac{\mathrm{d} f_{7}}{\mathrm{~d} p}-f_{9}-32 p \Omega_{0}\right)=0,
\end{aligned}
$$




$$
\begin{array}{r}
a_{1} a_{3}\left(\frac{\mathrm{d} f_{6}}{\mathrm{~d} p}-2 f_{10}-16 p \Omega_{1}+8 v\right)=0, \\
a_{1}^{2} a_{2}\left(G \Omega_{1}-f_{0} v\right)+2 a_{2} P_{0} \Omega_{0}-\frac{\mathrm{d} f_{8}}{\mathrm{~d} p}=0,
\end{array}
$$

$\frac{\partial R}{\partial \xi}=\mathscr{F}_{0}(p)+\mathscr{F}_{1}(p) \cos \xi+\mathscr{F}_{2}(p) \cos 2 \xi+\mathscr{F}_{3}(p) \cos 3 \xi+\mathscr{F}_{4}(p) \sin \xi+\mathscr{F}_{5}(p) \sin 2 \xi+\mathscr{F}_{6}(p) \sin 3 \xi$,

$$
\begin{aligned}
\frac{\partial R}{\partial p}= & \mathscr{F}_{7}(p) \cos \xi+\mathscr{F}_{8}(p) \cos 2 \xi+\mathscr{F}_{9}(p) \cos 3 \xi \\
& -a_{1}^{4}\left\{q_{3} m-\frac{\Omega_{1} f_{5}}{2}\right\} \cos 4 \xi+a_{3}\left\{a_{2}^{2} f_{7} \Omega_{0}+a_{1}^{2}\left(\frac{f_{6} \Omega_{1}}{2}+m q_{1}-\frac{1}{2} v q_{3}\right)\right\} \\
& \sin \xi-\frac{a_{1} a_{2} a_{3}}{16}\left\{v\left(q_{1} f_{0}+8 q_{2}\right)-8 f_{7} \Omega_{1}-16 f_{6} \Omega_{0}\right\} \sin 2 \xi+\frac{a_{3} a_{1}^{2}}{2}\left\{f_{6} \Omega_{1}+2 m q_{1}-v q_{3}\right\} \\
& \sin 3 \xi+\frac{a_{1}}{2} f_{3} \Omega_{1}-\frac{a_{3}^{2}}{2} q_{1} v+a_{2} f_{8} \Omega_{0}+a_{1}^{4} q_{3} m-\frac{a_{1}^{2} a_{2}^{2}}{16} q_{2} f_{0} v,
\end{aligned}
$$

$$
\begin{aligned}
& a_{1}^{4} a_{2}\left\{f_{0} f_{5} v-8 f q_{2} m \Omega_{1}-v f f_{13} \frac{\mathrm{d} f_{0}}{\mathrm{~d} p}-f_{0} f_{13} v \frac{\mathrm{d} f}{\mathrm{~d} p}-16 f q_{3} m \Omega_{0}-16 f_{4} m+f q_{3} \Omega_{1} f_{0} v+v f_{0} f_{5}\right. \\
& \left.\quad-2 f f_{0} v \frac{\mathrm{d} f_{13}}{\mathrm{~d} p}+8 f f_{12} \frac{\mathrm{d} m}{\mathrm{~d} p}+8 f_{12} m \frac{\mathrm{d} f}{\mathrm{~d} p}-f_{13} f_{0} f \frac{\mathrm{d} v}{\mathrm{~d} p}+16 f m \frac{\mathrm{d} f_{12}}{\mathrm{~d} p}\right\}=0, \\
& a_{3} a_{1}^{3}\left\{v f_{13} \frac{\mathrm{d} f}{\mathrm{~d} p}+f f_{13} \frac{\mathrm{d} v}{\mathrm{~d} p}-f f_{10} \frac{\mathrm{d} m}{\mathrm{~d} p}-2 f m \frac{\mathrm{d} f_{10}}{\mathrm{~d} p}-2 f_{6} m-f_{10} m \frac{\mathrm{d} f}{\mathrm{~d} p}-v f_{5}-f q_{3} \Omega_{1} v+2 f v \frac{\mathrm{d} f_{13}}{\mathrm{~d} p}+f q_{1} \Omega_{1} m\right\}=0, \\
& a_{1}\left\{-16 a_{1} m f_{3}+a_{1}^{2}\left(16 f_{13} f \frac{\mathrm{d} u}{\mathrm{~d} p}+16 f m \frac{\mathrm{d} f_{11}}{\mathrm{~d} p}+8 f_{11} f \frac{\mathrm{d} m}{\mathrm{~d} p}+8 f_{11} m \frac{\mathrm{d} f}{\mathrm{~d} p}-16 f q_{3} u \Omega_{1}+16 u f_{13} \frac{\mathrm{d} f}{\mathrm{~d} p}+32 f u \frac{\mathrm{d} f_{13}}{\mathrm{~d} p}\right)\right. \\
& \quad+a_{1}^{2} a_{2}^{2}\left(-f f_{0} f_{12} \frac{\mathrm{d} v}{\mathrm{~d} p}+f q_{2} f_{0} \Omega_{1} v+f_{4} v f_{0}-2 f f_{0} v \frac{\mathrm{d} f_{12}}{\mathrm{~d} p}+2 f q_{3} f_{0} \Omega_{0} v-v f f_{12} \frac{\mathrm{d} f_{0}}{\mathrm{~d} p}-16 f q_{2} \Omega_{0} m-f_{12} f_{0} v \frac{\mathrm{d} f}{\mathrm{~d} p}\right) \\
& \left.\quad+a_{3}^{2}\left(8 f f_{10} \frac{\mathrm{d} v}{\mathrm{~d} p}+8 f_{10} v \frac{\mathrm{d} f}{\mathrm{~d} p}+16 f v \frac{\mathrm{d} f_{10}}{\mathrm{~d} p}+8 f_{6} v-8 f q_{1} \Omega_{1} v\right)-8 a_{1}^{4} q_{3} f \Omega_{1} m\right\}=0,
\end{aligned}
$$

$a_{1}^{2} a_{2} a_{3}\left\{f_{10} f_{0} v \frac{\mathrm{d} f}{\mathrm{~d} p}+16 f q_{1} m \Omega_{0}-16 m f_{7}+f_{0} f_{6} v+f_{0} f_{10} f \frac{\mathrm{d} v}{\mathrm{~d} p}-16 f q_{3} v \Omega_{0}+8 f f_{12} \frac{\mathrm{d} v}{\mathrm{~d} p}+8 v f_{12} \frac{\mathrm{d} f}{\mathrm{~d} p}-8 f q_{2} v \Omega_{1}\right.$

$-8 f_{4} v+f f_{10} v \frac{\mathrm{d} f_{10}}{\mathrm{~d} p}-16 f m \frac{\mathrm{d} f_{9}}{\mathrm{~d} p}-8 f f_{9} \frac{\mathrm{d} m}{\mathrm{~d} p}+16 f v \frac{\mathrm{d} f_{12}}{\mathrm{~d} p}-8 f_{9} m \frac{\mathrm{d} f}{\mathrm{~d} p}-f q_{1} \Omega_{1} f_{0} v+2 f f_{0} v \frac{\mathrm{d} f_{10}}{\mathrm{~d} p}=0$,

$-2 a_{1}^{2} f_{8} m+\frac{a_{1} a_{2}}{16} f_{0} f_{3} v+\frac{a_{2} a_{1}^{4}}{16}\left\{-f f_{13} v \frac{\mathrm{d} v}{\mathrm{~d} p}-f f_{13} \frac{\mathrm{d} v}{\mathrm{~d} p}-2 f f_{0} v \frac{\mathrm{d} f_{13}}{\mathrm{~d} p}-f_{0} v f_{13} \frac{\mathrm{d} f}{\mathrm{~d} p}-f_{0} f_{5} v+2 f q_{3} \Omega_{1} f_{0} v\right\}$

$+a_{2} a_{1}^{2}\left(f f_{12} \frac{\mathrm{d} u}{\mathrm{~d} p}-\frac{1}{16} f f_{11} v \frac{\mathrm{d} f}{\mathrm{~d} p}+2 f u \frac{\mathrm{d} f_{12}}{\mathrm{~d} p}-f q_{2} u \Omega_{1}+f_{12} u \frac{\mathrm{d} f}{\mathrm{~d} p}-\frac{1}{16} f f_{11} f_{0} \frac{\mathrm{d} v}{\mathrm{~d} p}-\frac{1}{16} f f_{11} v \frac{\mathrm{d} f_{0}}{\mathrm{~d} p}-2 f q_{3} u \Omega_{0}-\frac{1}{8} f f_{0} v \frac{\mathrm{d} f_{11}}{\mathrm{~d} p}\right)$ $\left.+\frac{a_{2} a_{3}^{2}}{2}\left\{f_{7} v+2 f v \frac{\mathrm{d} f_{9}}{\mathrm{~d} p}-2 f q_{1} \Omega_{0} v+f_{9} f \frac{\mathrm{d} v}{\mathrm{~d} p}+f_{9} v \frac{\mathrm{d} f}{\mathrm{~d} p}\right\}+\frac{a_{1}^{2} a_{2}^{3}}{8} f q_{2} \Omega_{0} f_{0} v\right\}=0$, 


$$
\begin{aligned}
& a_{3} 8 f_{3} v+a_{1}\left(32 f u \frac{\mathrm{d} f_{10}}{\mathrm{~d} p}-16 f_{1} \Omega_{1} u-8 f_{11} v \frac{\mathrm{d} f}{\mathrm{~d} p}-8 f f_{11} \frac{\mathrm{d} v}{\mathrm{~d} p}+16 f_{10} u \frac{\mathrm{d} f}{\mathrm{~d} p}+16 f f_{10} \frac{\mathrm{d} u}{\mathrm{~d} p}-16 v f \frac{\mathrm{d} f_{11}}{\mathrm{~d} p}\right) \\
& +a_{1}^{3}\left(8 f_{13} v \frac{\mathrm{d} f}{\mathrm{~d} p}+16 v f \frac{\mathrm{d} f_{13}}{\mathrm{~d} p}+32 f m \frac{\mathrm{d} f_{10}}{\mathrm{~d} p}+16 m f_{10} \frac{\mathrm{d} f}{\mathrm{~d} p}+8 f_{5} v+16 f f_{10} \frac{\mathrm{d} m}{\mathrm{~d} p}-16 f q_{1} m \Omega_{1}+8 f_{13} f \frac{\mathrm{d} v}{\mathrm{~d} p}\right) \\
& \left.\quad+a_{1} a_{2}^{2}\left(2 f f_{0} q_{1} \Omega_{0} v-f f_{0} f_{9} \frac{\mathrm{d} v}{\mathrm{~d} p}-f_{0} f_{7} v-f_{9} f v \frac{\mathrm{d} f_{0}}{\mathrm{~d} p}-2 f_{0} f v \frac{\mathrm{d} f_{9}}{\mathrm{~d} p}+16 f q_{2} \Omega_{0} v\right)\right\}=0, \\
& a_{3}\left\{16 f_{8} v+a_{2}\left(16 f_{9} u \frac{\mathrm{d} f}{\mathrm{~d} p}+16 f_{9} f \frac{\mathrm{d} u}{\mathrm{~d} p}-32 f q_{1} \Omega_{0} u+32 f u \frac{\mathrm{d} f_{9}}{\mathrm{~d} p}\right)+a_{2} a_{1}^{2}\left(-8 f q_{2} v \Omega_{1}-3 f_{10} v f \frac{\mathrm{d} f_{0}}{\mathrm{~d} p}+8 f_{9} f \frac{\mathrm{d} m}{\mathrm{~d} p}+8 f_{4} v\right.\right. \\
& \quad-6 f f_{0} v \frac{\mathrm{d} f_{10}}{\mathrm{~d} p}-16 f q_{1} \Omega_{0} m-3 f_{0} f_{10} f \frac{\mathrm{d} v}{\mathrm{~d} p}+16 f v \frac{\mathrm{d} f_{12}}{\mathrm{~d} p}-3 f_{10} f_{0} v \frac{\mathrm{d} f}{\mathrm{~d} p}+3 f q_{1} \Omega_{1} f_{0} v+8 f_{12} f \frac{\mathrm{d} v}{\mathrm{~d} p}+f_{6} f_{0} v+8 f_{9} m \frac{\mathrm{d} f}{\mathrm{~d} p} \\
& \left.\left.\quad-16 f q_{3} \Omega_{0} v+8 f_{12} v \frac{\mathrm{d} f}{\mathrm{~d} p}+16 f m \frac{\mathrm{d} f_{9}}{\mathrm{~d} p}-16 f_{7} m\right)\right\}=0,
\end{aligned}
$$

$$
\begin{aligned}
& a_{1}\left\{16 f_{11} u \frac{\mathrm{d} f}{\mathrm{~d} p}+16 f_{11} f \frac{\mathrm{d} u}{\mathrm{~d} p}+32 f u \frac{\mathrm{d} f_{11}}{\mathrm{~d} p}-16 a_{1} f_{3} m+2 a_{2} f_{0} f_{8} v+a_{1}^{2}\left(-8 f_{11} m \frac{\mathrm{d} f}{\mathrm{~d} p}-16 f q_{3} u \Omega_{1}-16 f m \frac{\mathrm{d} f_{11}}{\mathrm{~d} p}-8 f_{11} f \frac{\mathrm{d} m}{\mathrm{~d} p}\right)\right. \\
& +a_{1}^{4}\left(8 f f_{13} \frac{\mathrm{d} f}{\mathrm{~d} p}+8 f_{13} m \frac{\mathrm{d} f}{\mathrm{~d} p}+16 f m \frac{\mathrm{d} f_{13}}{\mathrm{~d} p}\right)-32 a_{2}^{2} f q_{2} \Omega_{0} u+a_{3}^{2}\left(-8 f q_{1} \Omega_{1} v+8 f_{6} v+16 f v \frac{\mathrm{d} f_{10}}{\mathrm{~d} p}+8 f_{10} v \frac{\mathrm{d} f}{\mathrm{~d} p}+8 f f_{10} \frac{\mathrm{d} v}{\mathrm{~d} p}\right) \\
& \left.+a_{1}^{2} a_{2}^{2}\left(-f_{0} f_{12} f \frac{\mathrm{d} v}{\mathrm{~d} p}+f q_{2} \Omega_{1} f_{0} v+16 f q_{2} \Omega_{0} m-f_{0} v f_{12} \frac{\mathrm{d} f}{\mathrm{~d} p}-f_{4} f_{0} v-v f f_{12} \frac{\mathrm{d} f_{0}}{\mathrm{~d} p}-2 f f_{0} v \frac{\mathrm{d} f_{12}}{\mathrm{~d} p}+2 f q_{3} \Omega_{0} f_{0} v\right)\right\}=0,
\end{aligned}
$$

$$
\begin{aligned}
a_{3}\left\{a_{1}^{3}\left(f_{10} m \frac{\mathrm{d} f}{\mathrm{~d} p}+8 f_{10} f \frac{\mathrm{d} m}{\mathrm{~d} p}-8 f q_{1} \Omega_{1} m+16 f m \frac{\mathrm{d} f_{10}}{\mathrm{~d} p}-8 f q_{3} \Omega_{1} v-16 f_{6} m\right)+a_{1}\left\{a _ { 2 } ^ { 2 } \left(f_{7} f_{0} v-2 f f_{0} v \frac{\mathrm{d} f_{9}}{\mathrm{~d} p}-f_{0} f f_{9} \frac{\mathrm{d} v}{\mathrm{~d} p}\right.\right.\right. \\
\left.\quad+2 f q_{1} f_{0} v \Omega_{0}-16 f q_{2} \Omega_{0} v-f_{9} f v \frac{\mathrm{d} f_{0}}{\mathrm{~d} p}-f_{9} f_{0} v \frac{\mathrm{d} f}{\mathrm{~d} p}\right)+32 f u \frac{\mathrm{d} f_{10}}{\mathrm{~d} p}+8 f_{11} v \frac{\mathrm{d} f}{\mathrm{~d} p}+16 f v \frac{\mathrm{d} f_{11}}{\mathrm{~d} p}+16 f_{10} \times u \frac{\mathrm{d} f}{\mathrm{~d} p} \\
\left.\left.\quad-16 f q_{1} \Omega_{1} u+8 f_{11} f \frac{\mathrm{d} v}{\mathrm{~d} p}+16 f f_{10} \frac{\mathrm{d} u}{\mathrm{~d} p}\right\}+8 v f_{3}\right\}=0,
\end{aligned}
$$

where $\mathscr{F}_{i}(p), i=0,1, \ldots, 9$ is given in Appendix B. Systems (38)-(68) consist of thirty-two nonlinear ordinary differential equations in nineteen unknown functions. The solution to this system is somewhat intricate, and we cannot generally solve it as in the reversible case for arbitrary values of the parameters. But we solve it for certain values of the parameters leading to rigid body dynamics.

3.1. Two New Integrable Problems. The metric corresponding to the Lagrangian (9) that describes the metric of a rigid body matches with the Lagrangian (26) if we set

$$
\begin{gathered}
\xi=2\left(\varphi-\varphi_{0}\right), \\
p_{1}=p_{2}=p_{3}=1, \\
p=1+\frac{\gamma_{3}^{4}}{1-\gamma_{3}^{2}} .
\end{gathered}
$$

We are going to solve equation (70) taking into account condition (71). As a result of the complexity of those equations, we utilize the Maple program. We consider separately the two cases that are $a_{1} a_{2} \neq 0$ and $a_{2}=0$. Let us illustrate the causes of choosing those cases. In our works for constructing integrable systems with quartic integrals, it seems that some potentials are appropriate, with the attendance of a constant gyrostatic moment, while others are not. For more elucidation, we admit the Chaplygin case describing the motion of a rigid body in an incompressible ideal fluid and its generalization as an example:

$$
\begin{aligned}
& V_{1}=a\left(\gamma_{1}^{2}-\gamma_{2}^{2}\right)+2 b \gamma_{1} \gamma_{2}+\frac{\lambda}{2 \gamma_{3}^{2}}, \quad \boldsymbol{\mu}=(0,0, k), \\
& V_{2}=a\left(\gamma_{1}^{2}-\gamma_{2}^{2}\right)+2 b \gamma_{1} \gamma_{2}+\frac{\lambda}{2 \gamma_{3}^{2}}+\rho\left(\frac{1}{\gamma_{3}^{4}}-\frac{1}{\gamma_{3}^{6}}\right), \quad \boldsymbol{\mu}=(0,0,0),
\end{aligned}
$$


where $a, b, \lambda$, and $\rho$ are the arbitrary parameters, while $k$ is a constant characterizing the gyrostatic moment. These two cases were previously introduced in $[53,54]$, respectively. It is worth noticing that the singular term $\rho\left(\left(1 / \gamma_{3}^{4}\right)-\left(1 / \gamma_{3}^{6}\right)\right)$ is not compatible with the existence of the gyrostatic moment as it is outlined in (72), but in the absence of a gyrostatic moment, this term appears. As we see later, this situation appears, and it is followed by the discovery of two new cases. The new cases will be directly announced without any details due to most of the calculations cannot be displayed in a suitable size.

3.1.1. First New Integrable Case. We first consider the case in which $a_{2} a_{1} \neq 0$; taking into account the condition (71) and using the Maple program, we obtain a new integrable problem in a rigid body dynamic after tedious manipulations which are not writable in a suitable size in the generalized coordinates $\theta$ and $\varphi$. Therefore, we introduce it in the traditional Euler-Poisson variables for the sake of simplicity and to make the comparison clear with previous results.

Theorem 1. Let the principal inertia matrix for a rigid body satisfy the condition $A=B=2 C$, and the potential and gyroscopic forces characterized by Vand $\mu$, respectively, are given by

$$
\begin{aligned}
V= & \kappa\left[c\left(\gamma_{2}^{2}-\gamma_{1}^{2}\right)-2 d \gamma_{1} \gamma_{2}\right]+\frac{\lambda}{\gamma_{3}^{2}}+K\left[2 c \gamma_{1} \gamma_{2}+d\left(\gamma_{2}^{2}-\gamma_{1}^{2}\right)\right]\left(k-\nu \frac{\gamma_{1}^{2}+\gamma_{3}^{2}}{\gamma_{1}^{2}}\right)-\frac{k \nu \gamma_{3}^{2}}{\gamma_{1}^{2}}-\frac{\nu^{2} \gamma_{3}^{2}\left(\gamma_{3}^{2}+2 \gamma_{2}^{2}\right)}{2 \gamma_{1}^{4}} \\
& +K^{2}\left[\frac{d^{2}}{2}\left(\gamma_{3}^{4}+4 \gamma_{1}^{2} \gamma_{2}^{2}\right)-c^{2}\left(\gamma_{3}^{2}\left(\gamma_{1}^{2}+\gamma_{2}^{2}\right)+2 \gamma_{1}^{2} \gamma_{2}^{2}\right) 2+c d \gamma_{1} \gamma_{2}\left(\gamma_{1}^{2}-\gamma_{2}^{2}\right)\right], \\
\boldsymbol{\mu}= & \left(2 K \gamma_{3}\left(c \gamma_{2}-d \gamma_{1}\right)-\frac{2 \nu \gamma_{3}\left(1+\gamma_{2}^{2}\right)}{\gamma_{1}^{3}}, 2 K \gamma_{3}\left(c \gamma_{1}+d \gamma_{2}\right)+\frac{2 \nu \gamma_{2} \gamma_{3}}{\gamma_{1}^{2}}, k+K\left[2 c \gamma_{1} \gamma_{2}+d\left(\gamma_{2}^{2}-\gamma_{1}^{2}\right)\right]+\frac{\nu\left(1+\gamma_{2}^{2}\right)}{\gamma_{1}^{2}}\right),
\end{aligned}
$$

or, equivalently,

$$
\mathbf{l}=\left(0,0, k+K\left[2 c \gamma_{1} \gamma_{2}+d\left(\gamma_{2}^{2}-\gamma_{1}^{2}\right)\right]+\frac{\mathbf{v}\left(1+\gamma_{2}^{2}\right)}{\gamma_{1}^{2}}\right)
$$

$$
\begin{aligned}
I_{1}= & 2 p \gamma_{1}+2 q \gamma_{2}+\left(r+k+K\left(2 c \gamma_{1} \gamma_{2}+d\left(\gamma_{2}^{2}-\gamma_{1}^{2}\right)\right)\right. \\
& \left.+\frac{\nu\left(1+\gamma_{2}^{2}\right)}{\gamma_{1}^{2}}\right) \gamma_{3} .
\end{aligned}
$$

where $k, \kappa, c, d, \lambda, K$, and $\nu$ are the arbitrary parameters. Then, the Euler-Poisson equation 2) with (74) and (76) is integrable on a zero level of the area integral:
The additional integral admits the form

$$
\begin{aligned}
I_{2}= & {\left[p^{2}-q^{2}+c \kappa \gamma_{3}^{2}+c K^{2} \gamma_{3}^{2}\left(c\left(\gamma_{1}^{2}-\gamma_{2}^{2}\right)+2 d \gamma_{1} \gamma_{2}\right)-K d\left(2 \nu+2 k+\gamma_{3}^{2}(3 k-3 v-r)-\frac{\lambda\left(\gamma_{1}^{2}-\gamma_{2}^{2}\right)}{\gamma_{3}^{2}}\right)\right]^{2} } \\
& +\left[2 p q+d \kappa \gamma_{3}^{2}+d K^{2} \gamma_{3}^{2}\left(\left(\gamma_{1}^{2}-\gamma_{2}^{2}\right)+2 d \gamma_{1} \gamma_{2}\right)+c K\left(\gamma_{3}^{2}(3 k-3 v-r)-\frac{2 \lambda \gamma_{1} \gamma_{2}}{\gamma_{3}^{2}}\right)\right]^{2}+(k-\nu)(r-k+\nu) \\
& -K\left(2 c \gamma_{1} \gamma_{2}+d\left(\gamma_{2}^{2}-\gamma_{1}^{2}\right)\right)\left[2\left(p^{2}+q^{2}\right)\right]+2 \lambda\left(1+\frac{1}{\gamma_{3}^{2}}\right)+2 \gamma_{3}^{2}\left(\left(\gamma_{3}^{2}-1\right)\left(c^{2}+d^{2}\right) K^{2}-2 d(k-v) K+c \kappa\right) \\
& -4 \gamma_{3}(k-v)\left(2 K(k-v)\left[\left(c \gamma_{1}+2 d \gamma_{2}\right)+\kappa\left(d \gamma_{1}-2 c \gamma_{2}\right)\right] q+\gamma_{2} p(2 c(k-v) K+d \kappa)\right)
\end{aligned}
$$




$$
\begin{aligned}
& -8(k-v)\left[K c^{2}((k-v)) \times\left(\gamma_{3}^{4}-\gamma_{3}^{2}-\frac{1}{4}\right) K-\kappa \gamma_{1} \gamma_{2} \gamma_{3}^{2}+c\left[2(k-\nu) d K^{2} \gamma_{1} \gamma_{2} \gamma_{3}^{2}\right.\right. \\
& +K\left(\frac{d \kappa}{2} \gamma_{3}^{2}+d \kappa \gamma_{3}^{2}\left(\gamma_{1}^{2}-\frac{1}{2}\right)-2 \lambda \gamma_{1} \gamma_{2}\right)-\frac{\kappa(k-\nu)}{2} \gamma_{3}^{2}-\frac{(k-\nu) d^{2} K^{2}\left(16 \gamma_{1}^{2} \gamma_{3}^{2}+1\right)}{8}+\left(d\left((k-\nu)^{2}+\lambda\right) \gamma_{3}^{2}-2 d \lambda\left(\frac{1}{4}-\gamma_{1}^{2}\right)\right) K \\
& +\frac{K^{2} \nu \gamma_{3}^{4}}{\gamma_{1}^{2}}\left(c^{2}+d^{2}\right)\left[\frac{\nu}{\gamma_{1}^{2}}\right]\left(\gamma_{3}^{2}\left(2 \gamma_{1}^{2}+\gamma_{3}^{2}\right)\right)-2\left(\gamma_{1}^{2}+\gamma_{2}^{2}\right)-2 K\left(\gamma_{1}^{2}+\gamma_{2}^{2}\right)\left(2 c \gamma_{1} \gamma_{2}+d\left(\gamma_{2}^{2}-\gamma_{1}^{2}\right)+2\left(2 \gamma_{3}^{2}-1\right) r\right) \\
& +\frac{2 v^{2}\left(4 \gamma_{1}^{4}-\gamma_{3}^{2}\right)}{\gamma_{1}^{4}}\left(p^{2}-q^{2}\right)-\frac{2 k v \gamma_{3}^{2} r^{2}}{\gamma_{1}^{2}}+\frac{2 \gamma \gamma_{3}^{2}\left(p^{2}+q^{2}\right)}{\gamma_{1}^{2}}\left[2 K\left(c \gamma_{2}-d \gamma_{1}\right) \gamma_{1}-r\right] \\
& -\frac{4 \nu^{4} \gamma_{3}^{2}}{4 \gamma_{1}^{8}}\left[\gamma_{1}^{6}-\left(1+\gamma_{2}^{2}\right) \gamma_{4}^{4}+2\left(\gamma_{2}^{2}+1\right)^{2} \gamma_{1}^{2}+\gamma_{2}^{2} \gamma_{3}^{2}\left(\gamma_{2}^{2}+2\right)\right]+\frac{\nu}{\gamma_{1}^{6}} r \\
& \times\left[-2 v^{2}\left(\gamma_{1}^{2}\left(\gamma_{1}^{2}+3 \gamma_{2}^{2}\right)+\gamma_{3}^{2}\left(\gamma_{1}^{2}+\gamma_{2}^{2}\right)\right)-2 k v \gamma_{3}^{2} \gamma_{1}^{2}\left(\gamma_{1}^{2}-\gamma_{2}^{2}+2\right)-\gamma_{1}^{4}\left(2 \lambda\left(\gamma_{3}^{2}-1\right)+4 k K \gamma_{3}^{2}\left(2 c \gamma_{1} \gamma_{2}+d\left(\gamma_{1}^{2}+\gamma_{2}^{2}\right)\right)\right)\right. \\
& -\frac{2 \gamma^{3} \gamma_{3}^{2}}{\gamma_{1}^{6}}\left[k\left(\gamma_{1}^{2}\left(5 \gamma_{1}^{2}+4 \gamma_{3}^{2}-6\right)+\gamma_{3}^{2}\left(2-\gamma_{3}^{2}\right)\right)+K\left(\gamma_{2}\left(2 c \gamma_{1}\left(\gamma_{1}^{2}-\gamma_{2}^{2}\right)-d \gamma_{2}^{3}\right)\left(\gamma_{1}^{2}+\gamma_{3}^{2}\right)+d \gamma_{1}^{2}\left(\gamma_{1}^{2}+2 \gamma_{2}^{2}\right)\left(3 \gamma_{1}^{2}+\gamma_{3}^{2}\right)\right)\right. \\
& +\frac{2 \nu \lambda}{\gamma_{1}^{4}}\left[\nu\left(\gamma_{2}^{2}-\gamma_{1}^{2}\right)+\gamma_{1}^{2}\left(k+K\left(2 c d \gamma_{1} \gamma_{2}+d\left(\gamma_{2}^{2}-\gamma_{1}^{2}\right)\right)\right)\right]-\frac{2 \nu \gamma_{3}^{2} \kappa}{\gamma_{1}^{4}}\left[\nu \gamma_{2}\left(\gamma_{2}\left(4 \gamma_{1}^{4}+\gamma_{3}^{2}\right)\right)-2 d \gamma_{1}\left(2 \gamma_{1}^{2}+\gamma_{3}^{2}\right)+\gamma_{1}^{2}\left(2 \gamma_{3}\right)\right. \\
& \times\left(c \gamma_{2}-d \gamma_{1}\right) q-2 \gamma_{3}\left(c \gamma_{1}+d \gamma_{2}\right) p+k\left(4 d \gamma_{1} \gamma_{2}-4\right)\left(\gamma_{3}^{2}+4 \gamma_{2}^{2}\right)+2 v^{2}\left[-5 K d k \gamma_{3}^{6}-4\left(\left(2 c q-\frac{1}{2} d p\right) \gamma_{1}+\gamma_{2}\left(c p+\frac{3}{2} d q\right)\right)\right. \\
& \times K \gamma_{3}^{5}+2\left(k\left(\left(5-8 \gamma_{1}^{2}\right) d+5 c \gamma_{1} \gamma_{2}\right) K-k^{2}-2 q^{2}\right) \gamma_{3}^{2}-4\left(5 c \gamma_{1}^{3} q+\gamma_{2}(c p+4 d q) \gamma_{1}^{2}+\gamma_{1}\left(\frac{d p}{2}-2 c q\right)-\gamma_{2}\left(c p+\frac{3 d q}{2}\right)\right) \\
& \times K \gamma_{3}^{3}+\gamma_{3}^{2}\left(k\left(\left(16 \gamma_{1}^{2}-8 \gamma_{1}^{-} 5\right) d+2 \gamma_{1} \gamma_{2}\left(6 \gamma_{1}^{2}-5\right) c\right) K+\gamma_{1}^{2}\left(2 p^{2}-6 q^{2}-4 k^{2}\right)+4 \gamma_{1} \gamma_{2} p q+k^{2}\right)+8 K\left((-c q-d p) \gamma_{1}^{3}\right) \\
& \left.\gamma_{2}(c p-d q) \gamma_{1}^{2}+\frac{3}{2} c q \gamma_{1}+\frac{\gamma_{2}}{2}(c p+2 d q) \gamma_{3} \gamma_{1}^{2}+4 q \gamma_{1}^{2}\left(2 \gamma_{1} \gamma_{2} p+q\right)\right]+\frac{4 \nu}{\gamma_{1}^{2}}\left[-\frac{1}{2} k K^{2} \gamma_{3}^{2}\left(c^{2}+2 d^{2}\right)+\gamma_{3}^{4}\left(\left(2 c k \gamma_{1}\right)\right)\right. \\
& \times\left(c \gamma_{1}+d \gamma_{2}\right)+d^{2} k K^{2}+\left(\left(3 k^{2}+p^{2}-2 q^{2}\right) d-3 p q c\right) K+2 k\left(\left(3 q \gamma_{2}-p \gamma_{1}\right)\right) d+c\left(2 q \gamma_{1}+\gamma_{2} q\right) K \gamma_{3}^{3}+\left(2 k\left(\left(\gamma_{1}^{4}\right)\right)\right) \\
& -c d \gamma_{1} \gamma_{2}-c^{2} \gamma_{1}^{2}\left(\gamma_{2}^{2}+\gamma_{3}^{2}\right) K^{2}+\gamma_{3}^{2}\left(\left(2 k^{2}\left(\gamma_{1}^{2}-2\right)-\frac{1}{2} p^{2}+\frac{3}{2} q^{2}\right) d+2 c\left(p q-2 k^{2} \gamma_{1}^{2} \gamma_{2}^{2}\right)\right) K+\frac{k^{3}}{2} \\
& -4 K k\left(d q \gamma_{2}+c\left(\gamma_{1}^{2}\left(\gamma_{2} p-\gamma_{1} q\right)+\frac{1}{2}\left(\gamma_{2} p+3 \gamma_{1} q\right)\right) \gamma_{3}\right)
\end{aligned}
$$

Theorem 1 characterizes a new integrable problem in a rigid body dynamics. The present case generalizes a special version of the case introduced by Yehia and Elmandouh in 2016 by adding a new parameter $v$ [48]. Also, it includes the case announced by Elmandouh in $2015(K=0)$ [3]. Moreover, it generalizes the case presented by Yehia and Elmandouh in 2013 by inserting two arbitrary constants $(k=\nu=0)$ [6]. It generalizes the integrable case which was introduced by Goriatchev in 1916 by adding four arbitrary parameters $\nu, k, K$, and $\rho$ [55]. It also contains five arbitrary parameters, $\nu, k, K, \lambda$, and $\rho$, more than the case introduced by Chaplygin in 1903 [56]. To avoid confusion, we summed up the comparisons between this case and the related earlier cases in Table 2.

Regrettably, the physical interpretation for the whole system with the full set of all associated parameters is unknown. Disregarding the singular terms in both potential and vector functions, the problem describes physically the 
TABLE 2: Comparison the first integrable case with previous results.

\begin{tabular}{lccc}
\hline No. & Authors & Conditions of the parameters & References \\
\hline 1 & Yehia and Elmandouh [48] & $\nu=0$ & {$[48]$} \\
2 & Elmandouh [3] & $K=0$ & {$[3]$} \\
3 & Yehia and Elmandouh [6] & $v=\nu=0$ & {$[6]$} \\
4 & Goriatchev [55] & $\nu=K=\rho=k=0$ & {$[55]$} \\
5 & Chaplygin [56] & $\nu=K=\rho=k=\lambda=0$ & {$[56]$} \\
\hline
\end{tabular}

motion of an electrically charged heavy rigid body about a fixed point under the action of potential and gyroscopic forces admitting a common axis of symmetry [6].

3.2. Second New Integrable Problem. In this subsection, we solve the basic equations in the case in which $a_{2}=0$ taking into account the conditions.
Theorem 2. Let the inertia matrix of a rigid body be $I=\operatorname{diag}(2 \mathrm{C}, 2 \mathrm{C}, \mathrm{C})$, and assume this body be in motion under the action of a combination of following potential and gyroscopic forces which are characterized by Vand $\mu$, respectively,

$$
\begin{aligned}
V= & \kappa\left[2 d \gamma_{1} \gamma_{2}+c\left(\gamma_{1}^{2}-\gamma_{2}^{2}\right)\right]+\frac{\lambda}{\gamma_{3}^{2}}+\rho\left(\frac{1}{\gamma_{3}^{4}}-\frac{1}{\gamma_{3}^{6}}\right)-\frac{K \nu \gamma_{3}^{2}}{\gamma_{1}^{2}}\left[2 c \gamma_{1} \gamma_{2}+d\left(\gamma_{2}^{2}-\gamma_{1}^{2}\right)\right]+\frac{\nu^{2} \gamma_{3}^{2}\left(\gamma_{3}^{2}-2\right)}{2 \gamma_{1}^{2}} \\
& +K^{2}\left[2 d c \gamma_{1} \gamma_{2}\left(\gamma_{1}^{2}-\gamma_{2}^{2}\right)+\frac{d^{2}}{2}\left(\gamma_{3}^{2}+4 \gamma_{1}^{2} \gamma_{2}^{2}\right)-c^{2}\left(\gamma_{3}^{2}\left(\gamma_{1}^{2}+\gamma_{2}^{2}\right)+2 \gamma_{1}^{2} \gamma_{2}^{2}\right)\right], \\
\boldsymbol{\mu}= & \left(2 \gamma_{3}\right)\left(K\left(c \gamma_{2}-d \gamma_{1}\right)-\frac{\nu\left(1+\gamma_{2}^{2}\right)}{g_{1}^{3}}\right), 2 \gamma_{3}\left(K\left(c \gamma_{1}+d \gamma_{2}\right)+\frac{v \gamma_{2}^{2}}{\gamma_{1}^{2}}\right), K\left(2 c \gamma_{1} \gamma_{2}+d\left(\gamma_{2}^{2}-\gamma_{1}^{2}\right)+v \frac{\left(2-\gamma_{3}^{2}\right)}{\gamma_{1}^{2}}\right),
\end{aligned}
$$

or, equivalently,

$$
I_{1}=2\left(p \gamma_{1}+q \gamma_{2}\right)+\left(r+K\left[2 c \gamma_{1} \gamma_{2}+d\left(\gamma_{2}^{2}-\gamma_{1}^{2}\right]+v \frac{\left(2-\gamma_{3}^{2}\right)}{\gamma_{1}^{2}} \gamma_{3}\right]\right. \text {. }
$$

$$
\mathbf{l}=\left(\mathbf{0}, \mathbf{0}, K\left[2 c \gamma_{1} \gamma_{2}+d\left(\gamma_{2}^{2}-\gamma_{1}^{2}\right]+v \frac{\left(2-\gamma_{3}^{2}\right)}{\gamma_{1}^{2}}\right)\right),
$$

where $c, d, \kappa, K, \lambda, \rho$, and $\nu$ are the arbitrary parameters. Then, the Euler-Poisson equation (2) with the two expressions (79) and (80) is completely integrable on a zero level of the cyclic integral:

Its additional integral takes the form

$$
\begin{aligned}
I_{2}= & {\left[p^{2}-q^{2}+\gamma_{3}^{2}(K d r+c \kappa)+c K^{2} \gamma_{3}^{2}\left(c\left(\gamma_{1}^{2}-\gamma_{2}^{2}\right)+2 d \gamma_{1} \gamma_{2}\right)-\lambda \frac{\left(\gamma_{1}^{2}-\gamma_{2}^{2}\right)}{\gamma_{3}^{2}}\right]^{2} } \\
& +\left[2 p q+d \kappa \gamma_{3}^{2}-\gamma_{3}^{2}\left[K c r-d K^{2}\left(c\left(\gamma_{1}^{2}-\gamma_{2}^{2}\right)+2 d \gamma_{1} \gamma_{2}\right)-\frac{2 \lambda \gamma_{1}^{2} \gamma_{2}^{2}}{\gamma_{3}^{2}}-d K^{2}\left(c\left(\gamma_{1}^{2}-\gamma_{2}^{2}\right)+2 d \gamma_{1} \gamma_{2}\right)-\frac{2 \lambda \gamma_{1}^{2} \gamma_{2}^{2}}{\gamma_{3}^{2}}\right]^{2}\right. \\
& -\frac{\nu^{2}\left(\gamma_{1}^{2}+\gamma_{2}^{2}\right)^{2}}{\gamma_{1}^{8} \gamma_{3}^{4}}\left[2 \gamma_{1}^{4}\left(\rho+\lambda \gamma_{3}^{4}\right)-\nu^{2} \gamma_{3}^{2}\right]+\frac{4 \nu\left(p \gamma_{1}+q \gamma_{2}\right)}{\gamma_{1}^{2}} \times\left[\frac{\nu^{2} \gamma_{3}^{3}\left(\gamma_{1}^{2}+\gamma_{1}^{2}\right)}{\gamma_{1}^{4}}+\frac{\left(\gamma_{3}^{2}-1\right)\left(\gamma_{3}^{4} \lambda+\rho\right)}{\gamma_{3}^{5}}-2 K^{2}\left(c^{2}+d^{2}\right)\left(2 \gamma_{3}^{2}-1\right) \gamma_{3}^{3}\right] \\
& +2 K\left[2 c \gamma_{1} \gamma_{2}+d\left(\gamma_{2}^{2}-\gamma_{1}^{2}\right)\right]
\end{aligned}
$$


TABLE 3: Comparison the second new integrable case with previous results.

\begin{tabular}{lccc}
\hline No. & Authors & Conditions of the parameters & References \\
\hline 1 & Yehia and Elmandouh [6] & $\nu=0$ & {$[6]$} \\
2 & Goriatchev [55] & $K=\nu=\rho=0$ & {$[55]$} \\
3 & Chaplygin [56] & $K=\nu=\lambda=\rho=0$ & {$[56]$} \\
\hline
\end{tabular}

$$
\begin{aligned}
& \times\left[-\frac{\nu K^{2} \gamma_{3}^{6}\left(c^{2}+d^{2}\right)}{\gamma_{1}^{2}}-\frac{\rho r}{\gamma_{3}^{4}}+\frac{\nu\left(\lambda \gamma_{3}^{4}-\rho\right)}{\gamma_{1}^{2} \gamma_{3}^{2}}+\frac{2 \nu^{3} \gamma_{3}^{4}\left(\gamma_{1}^{2}+\gamma_{2}^{2}\right)}{\gamma_{1}^{6}}\right]+2\left(\frac{\rho \kappa}{\gamma_{3}^{4}}+\frac{\nu^{2} \gamma_{3}^{4}}{\gamma_{1}^{4}}\right)\left[2 d \gamma_{1} \gamma_{2}+c\left(\gamma_{1}^{2}-\gamma_{2}^{2}\right)\right] \\
& +2\left(p^{2}+q^{2}\right)\left[\frac{\rho\left(\gamma_{3}^{2}-1\right)}{\gamma_{3}^{6}}+\frac{2 v \gamma_{3}\left(p \gamma_{1}+q \gamma_{2}\right)}{\gamma_{1}^{2}}+\frac{4 \nu K \gamma_{3}^{2}\left(c \gamma_{2}-d \gamma_{1}\right)}{\gamma_{1}}\right] \\
& +\rho\left[\frac{\left(\rho-2 \lambda \gamma_{3}^{4}\right)\left(1-\gamma_{3}^{2}\right)^{2}}{\gamma_{3}^{12}}+\frac{2 K^{2}}{\gamma_{3}^{4}}\left[c^{2}\left(1-2 \gamma_{3}^{2}\right)-4 \gamma_{1} \gamma_{2}\left(c \gamma_{2}-d \gamma_{1}\right)\left(c \gamma_{1}+d \gamma_{2}\right)\right]\right] \\
& +\frac{\nu^{2} \gamma_{3}^{2}}{\gamma_{1}^{4}}\left[K^{2}\left(c^{2}+d^{2}\right) \gamma_{3}^{2}\left(5 \gamma_{3}^{2}-8 \gamma_{3}^{2}+2\right)+4 K \gamma_{3}\left(\gamma_{1}^{2}+\gamma_{2}^{2}\right) \times\left((4 c q-d p) \gamma_{1}+\gamma_{2}(2 c p+3 d q)\right)\right. \\
& \left.-2 \gamma_{1}^{2}\left[4 K \gamma_{3}\left(\left(c \gamma_{1}+d \gamma_{2}\right) q+\left(d \gamma_{1}-c \gamma_{2}\right) p\right)+2\left(q^{2}-p^{2}\right)-\left(\gamma_{1}^{2}+\gamma_{2}^{2}\right) \times\left(p^{2}+3 q^{2}\right)-4 p q \gamma_{1} \gamma_{2}\right]\right] \\
& -\frac{2 v \gamma_{3}^{2}}{\gamma_{1}^{4}}\left[K \gamma_{3}^{2}\left(6 c p q+d\left(5 q^{2}-p^{2}\right)\right)+2(c p+d q)\left(\kappa \gamma_{1} \gamma_{3}+2 K q\right)(c p+d q)-4 \kappa \gamma_{2} \gamma_{3}(c q-d p)\right] .
\end{aligned}
$$

Theorem 2 introduces a new integrable problem in the dynamics of a rigid body. Furthermore, it represents an extension for the related previous results. It adds to the case that was discovered by Yehia and Elmandouh in 2013, one arbitrary parameter $v$ [6]. It modifies the case introduced by Goriatchev in 1916 by inserting three parameters $K, v$, and $\rho$ [55]. It generalizes the Chaplygin case that was found in 1903 by entering four arbitrary parameters $\nu, \rho, \lambda$, and $K$ [56]. The comparisons with previous results are summarized in Table 3.

\section{Conclusion}

In the current work, we had interest in studying the integrability issue of the motion of a rigid body about a fixed point under the action of potential and gyroscopic forces having a common axis of symmetry. We have assumed this problem has a complementary quartic integral in the velocities. We have applied the method by Yehia. The basic equations have been formulated and introduced in a general setting. But as it is outlined in the literature, in the case of the existence of gyroscopic forces, the basic equations have not been solved in a general setting, but it is usually solved for certain values of the parameters leading to the metric of a rigid body dynamics which are valuable and significant problems. We have announced two new integrable problems generalizing the Chaplygin case in a rigid body and its subsequent works by different authors such as Goriatchev, Yehia and Elmandouh, and Elmandouh. The comparison of new results with previous ones is summarized and collected in Tables 2 and 3.

\section{Appendix}

\section{A. Time Transformation}

Consider Lagrangian in the form

$$
L:=\frac{\Lambda}{2}\left(\dot{x}^{2}+\dot{y}^{2}\right)+l_{1} \dot{x}+l_{2} \dot{y}-V,
$$

where $\Lambda, l_{1}, l_{2}$, and $V$ are the functions in $x$ and $y$ variables. Lagrangian (A.1) has a Jacobi integral in the form

$$
I_{1}=\frac{\Lambda}{2}\left(\dot{x}^{2}+\dot{y}^{2}\right)+V=h,
$$

where $h$ is the value of the Jacobi integral. Performing the time transformation,

$$
\mathrm{d} t=\Lambda \mathrm{d} \tau
$$

to the Lagrangian (A.1), we obtain

$$
L_{0}=\frac{1}{2}\left(x^{\prime^{2}}+y^{\prime^{2}}\right)+l_{1} x^{\prime}+l_{2} y^{\prime}+\Lambda(h-V),
$$

where dash refers to the derivative with respect to the fictitious time $\tau$. The Lagrangian (A.4) has a Jacobi integral in the form

$$
I_{2}=\frac{1}{2}\left(x^{\prime^{2}}+y^{\prime^{2}}\right)-\Lambda(h-V)=h^{\prime}
$$

where $h^{\prime}$ is the value of the Jacobi integral for Lagrangian (A.5). Doing the inverse of the time transformation (A.2) to the Jacobi integral (A.5), we get 


$$
I_{3}=\frac{\Lambda}{2}\left(\dot{x}^{2}+\dot{y}^{2}\right)+V=\frac{h \prime}{V}+h .
$$

The two integrals of the motion (A.3) and (A.6) are identical if $h \prime=0$. Thus, the two Lagrangian $L$ and $L_{0}$ are equivalent on the zero level of the Jacobi integral for the second one.

\section{B. Coefficients of Equations (60) and (61)}

$$
\begin{aligned}
& \mathscr{F}_{0}(p)=-\frac{1}{2} a_{1}^{4}\left\{f_{13} f \Omega_{1}+q_{3} m \frac{\mathrm{d} f}{\mathrm{~d} p}+2 f q_{3} m-4 T m+q_{3} f \frac{\mathrm{d} m}{\mathrm{~d} p}-16 f m \Omega_{1}\right\} \sin 4 \xi \\
& +\frac{a_{1} a_{2} a_{3}}{16}\left\{2 f f_{0} v \frac{\mathrm{d} q_{1}}{\mathrm{~d} p}+q_{1} f_{0} f \frac{\mathrm{d} v}{\mathrm{~d} p}-16 f f_{10} \Omega_{0}-16 G v-8 q_{2} f \frac{\mathrm{d} v}{\mathrm{~d} p}+256 f v \Omega_{0}-8 f f_{9} \Omega_{1}-16 f v \frac{\mathrm{d} q_{2}}{\mathrm{~d} p}-8 q_{2} v \frac{\mathrm{d} f}{\mathrm{~d} p}\right. \\
& \left.+q_{1} v f \frac{\mathrm{d} f_{0}}{\mathrm{~d} p}-32 p f_{0} v+q_{1} f_{0} v \frac{\mathrm{d} f}{\mathrm{~d} p}\right\} \\
& \mathscr{F}_{1}(p)=\frac{1}{2} a_{3}\left\{-4 f u \frac{\mathrm{d} q_{1}}{\mathrm{~d} p}-2 q_{1} u \frac{\mathrm{d} f}{\mathrm{~d} p}-4 v P_{0}-2 q_{1} f \frac{\mathrm{d} u}{\mathrm{~d} p}+a_{1}^{2}\left(16 f v \Omega_{1}+64 p m-2 T v-q_{1} f \frac{\mathrm{d} m}{\mathrm{~d} p}-2 f m \frac{\mathrm{d} q_{1}}{\mathrm{~d} p}-2 f v \frac{\mathrm{d} q_{3}}{\mathrm{~d} p}\right.\right. \\
& \left.\left.-q_{3} f \frac{\mathrm{d} v}{\mathrm{~d} p}-q_{1} m \frac{\mathrm{d} f}{\mathrm{~d} p}-q_{3} v \frac{\mathrm{d} f}{\mathrm{~d} p}-2 f f_{10} \Omega_{1}\right)-2 a_{2}^{2} f f_{9} \Omega_{0}\right\}, \\
& \mathscr{F}_{2}(p)=-\frac{a_{1} a_{2} a_{3}}{16}\left\{16 v G-q_{1} f f_{0} \frac{\mathrm{d} v}{\mathrm{~d} p}-8 q_{2} f \frac{\mathrm{d} v}{\mathrm{~d} p}-2 f f_{0} v \frac{\mathrm{d} q_{1}}{\mathrm{~d} p}-q_{1} v f_{0} \frac{\mathrm{d} f}{\mathrm{~d} p}-q_{1} v f \frac{\mathrm{d} f_{0}}{\mathrm{~d} p}-8 q_{2} v \frac{\mathrm{d} f}{\mathrm{~d} p}-32 p f_{0} v+16 f_{10} f \Omega_{0}\right. \\
& \left.+256 v \Omega_{0}-16 v f \frac{\mathrm{d} q_{2}}{\mathrm{~d} p}+8 f f_{9} \Omega_{1}\right\} \\
& \mathscr{F}_{3}(p)=-\frac{a_{1} a_{2}}{16}\left\{-a_{1}^{2} q_{3} f_{0} v \frac{\mathrm{d} f}{\mathrm{~d} p}+2 f f_{0} v \frac{\mathrm{d} q_{3}}{\mathrm{~d} p}+32 G m+q_{3} f f_{0} \frac{\mathrm{d} v}{\mathrm{~d} p}-16 f f_{0} v \Omega_{1}+2 T v\right\}, \\
& \mathscr{F}_{4}(p)=-\frac{1}{2} a_{1}^{2} a_{3}\left\{2 f m \frac{\mathrm{d} q_{1}}{\mathrm{~d} p}+64 p m-q_{3} v \frac{\mathrm{d} f}{\mathrm{~d} p}+q_{1} f \frac{\mathrm{d} m}{\mathrm{~d} p}+f f_{10} \Omega_{1}-2 f v \frac{\mathrm{d} q_{3}}{\mathrm{~d} p}-q_{3} f \frac{\mathrm{d} v}{\mathrm{~d} p}+16 f v \Omega_{1}+q_{1} m f \frac{\mathrm{d} f}{\mathrm{~d} p}\right. \\
& \left.+2 T f_{0} v-16 q_{2} u \frac{\mathrm{d} f}{\mathrm{~d} p}-8 f f_{12} \Omega_{1}+8 q_{2} f \frac{\mathrm{d} m}{\mathrm{~d} p}-16 f q_{2} \frac{\mathrm{d} u}{\mathrm{~d} p}+16 f m \frac{\mathrm{d} q_{2}}{\mathrm{~d} p}+q_{3} v f \frac{\mathrm{d} f_{0}}{\mathrm{~d} p}+8 q_{2} m \frac{\mathrm{d} f}{\mathrm{~d} p}-256 f m \Omega_{0}\right\} \\
& +16 q_{2} f \frac{\mathrm{d} u}{\mathrm{~d} p}+16 f f_{11} \Omega_{0}-512 f u \Omega_{0}+32 f u \frac{\mathrm{d} q_{2}}{\mathrm{~d} p} \\
& \mathscr{F}_{5}(p)=-\frac{1}{16}\left\{a_{1}^{2}\left(16 q_{3} f \frac{\mathrm{d} u}{\mathrm{~d} p}+8 f_{11} f \Omega_{1}+16 q_{3} u \frac{\mathrm{d} f}{\mathrm{~d} p}-64 P_{0} m-256 f u \Omega_{1}+32 f u \frac{\mathrm{d} q_{3}}{\mathrm{~d} p}\right)\right. \\
& +a_{3}^{2}\left(8 q_{1} v \frac{\mathrm{d} f}{\mathrm{~d} p}+16 f v \frac{\mathrm{d} q_{1}}{\mathrm{~d} p}+256 p v+8 q_{1} f \frac{\mathrm{d} v}{\mathrm{~d} p}\right)+a_{1}^{2} a_{2}^{2}\left(16 f f_{12} \Omega_{0}-q_{2} f_{0} f \frac{\mathrm{d} v}{\mathrm{~d} p}+2 G v f_{0}-2 f f_{0} v \frac{\mathrm{d} q_{2}}{\mathrm{~d} p}\right. \\
& \left.\left.+32 f f_{0} v \Omega_{0}-q_{2} f_{0} v \frac{\mathrm{d} f}{\mathrm{~d} p}-q_{2} v f \frac{\mathrm{d} f_{0}}{\mathrm{~d} p}\right)+8 a_{1}^{4} f f_{13} \Omega_{1}\right\} \\
& \mathscr{F}_{6}(p)=-\frac{a_{2} a_{1}^{3}}{16}\left\{-q_{3} f_{0} v \frac{\mathrm{d} f}{\mathrm{~d} p}+8 q_{2} m \frac{\mathrm{d} f}{\mathrm{~d} p}-256 f m \Omega_{0}+16 f f_{13} \Omega_{0}+8 q_{2} f \frac{\mathrm{d} m}{\mathrm{~d} p}+16 f f_{0} v \Omega_{1}+16 f m \frac{\mathrm{d} q_{2}}{\mathrm{~d} p}-32 G m-q_{3} f_{0} f \frac{\mathrm{d} v}{\mathrm{~d} p}\right. \\
& \left.+2 T f_{0} v+8 f_{12} f \Omega_{1}-q_{3} v f \frac{\mathrm{d} f_{0}}{\mathrm{~d} p}-2 f f_{0} v \frac{\mathrm{d} q_{3}}{\mathrm{~d} p}\right\} \\
& \mathscr{F}_{7}(p)=\frac{a_{1}}{2}\left\{\left(2 f_{8}+a_{1}^{2} a_{2} f_{4}\right) \Omega_{1}+\left(f_{3} \Omega_{0}+a_{2} a_{1}^{3}\left(q_{2} m-\frac{1}{16} q_{3} f_{0} v\right)\right)\right\} \\
& \mathscr{F}_{8}(p)=\frac{1}{16}\left\{8 a_{1}^{4} f_{5} \Omega_{1}+a_{1}^{2} a_{2}^{2} q_{2} f_{0} v+16 a_{1}^{2} a_{2}^{2} f_{4} \Omega_{0}-8 a_{3}^{2} q_{1} v+8 a_{1} f_{3} \Omega_{1}\right\}, \\
& \mathscr{F}_{9}(p)=-a_{2} a_{1}^{3}\left\{q_{2} m-\frac{1}{2} f_{4} \Omega_{1}-\frac{1}{16} q_{3} f_{0} v-f_{5} \Omega_{0}\right\} .
\end{aligned}
$$




\section{Data Availability}

No data were used to support this study.

\section{Conflicts of Interest}

The authors declare that they have no conflicts of interest.

\section{Acknowledgments}

The authors acknowledge the Deanship of Scientific Research at King Faisal University for the financial support under the annual research project (Grant No. 180100).

\section{References}

[1] O. Babelon, D. Bernard, and M. Talon, Introduction to Classical Integrable Systems, Cambridge University Press, Cambridge, UK, 2003.

[2] M. . Tabor, Chaos and Integrability in Nonlinear Dynamics, Wiley, NewYork, NY, USA, 1988.

[3] A. A. Elmandouh, "New integrable problems in rigid body dynamics with quartic integrals," Acta Mechancia, vol. 226, Article ID 246172, 2015.

[4] A. A. Elmandouh, "New integrable problems in the dynamics of particle and rigid body," Acta Mechancia, vol. 226, pp. 2461-2472, Article ID 374962, 2015.

[5] H. M. Yehia, "Atlas of two-dimensional irreversible conservative lagrangian mechanical systems with a second quadratic integral," Journal of Mathematical Physics, vol. 48, Article ID 082902, 2007.

[6] H. M. Yehia and A. A. Elmandouh, "A new integrable problem with a quartic integral in the Dynamics of a rigid body," Journal of Physics A: Mathematical and Theoretical, vol. 46, no. 14, Article ID 142001, 2013.

[7] M. Karlovini, G. Pucacco, K. Rosquist, and L. Samuelsson, “A unified treatment of quartic invariants at fixed and arbitrary energy," Journal of Mathematical Physics, vol. 43, Article ID 404159, 2002.

[8] Z. Hu, M. Aldazharova, T. M. Aldibekov, and V. G. Romanovski, "Integrability of 3-dim polynomial systems with three invariant planes," Nonlinear Dynamics, vol. 74, Article ID 107792, 2013.

[9] J. Llibre, R. Ramirez, and N. Sadovskaia, "Integrability of the constrained rigid body," Nonlinear Dynamics, vol. 73, Article ID 227390, 2013.

[10] J. Llibre, R. D. Oliveira, and C. Valls, "On the integrability and the zero-hopf bifurcation of a Chen-Wang differential system," Nonlinear Dynamics, vol. 80, p. 35361, 2015.

[11] J. Bao and Q. Yang, "Darboux integrability of the stretchtwistfold flow," Nonlinear Dynamics, vol. 76, Article ID 797807, 2014.

[12] M. F. Lima, J. Llibre, and C. Valls, "Integrability of the rucklidge system," Nonlinear Dynamics, vol. 77, Article ID 144153, 2014.

[13] A. A. Elmandouh, "First integrals of motion for two dimensional weight-homogeneous Hamiltonian systems in curved spaces," Communications in Nonlinear Science and Numerical Simulation, vol. 75, pp. 220-235, 2019.

[14] T. Bountis, H. Segur, and F. Vivaldi, "Integrable hamiltonian systems and the painlevé property," Physical Review A, vol. 25, no. 3, p. 1257, 1982.
[15] S. L. V. Ziglin, "Branching of solutions and nonexistence of first integrals in hamiltonian mechanics," Functional Analysis and Its Applications, vol. 16, p. 1819, 1982.

[16] J. J. Morales-Ruiz, Differential Galois Theory and Non-integrability of Hamiltonian Systems, Prog. Math. Birkhauser Verlag, Basel, Switzerland, 1999.

[17] J. J. Morales-Ruiz and J. P. Ramis, "A note on the nonintegrability of some hamiltonian systems with a homogeneous potential," Methods and Applications of Analysis, vol. 8, p. 11320, 2001.

[18] J. J. Morales-Ruiz and J. P. Ramis, "Galoisian obstructions to integrability of hamiltonian systems," Methods and Applications of Analysis, vol. 8, p. 3396, 2001.

[19] A. A. Elmandouh, "On the integrability of 2D hamiltonian systems with variable gaussian curvature," Nonlinear Dynamics, vol. 93, p. 93343, 2018.

[20] A. A. Elmandouh, "On the integrability of new examples of two-dimensional hamiltonian systems in curved spaces," Communications in Nonlinear Science and Numerical Simulation, vol. 90, Article ID 105368, 2020.

[21] V. Volterra, "Sur la théorie des variations des latitudes," Acta Mathematica, vol. 22, pp. 201-357, 1899.

[22] A. Gluhovsky and C. Tong, "The structure of energy conserving low-order models," Physics of Fluids, vol. 11, no. 2, pp. 334-343, 1999.

[23] P. C. Hughes, Spacecraft Attitude Dynamics, Wiely, New york, NY, USA, 1986.

[24] T. Amer and W. Amer, "Substantial condition for the fourth first integral of the rigid body problem," Mathematics and Mechanics of Solids, vol. 23, no. 8, pp. 1237-1246, 2018.

[25] T. S. Amer, A. M. Farag, and W. S. Amer, "The dynamicsamical motion of a rigid body for the case of ellipsoid inertia close to ellipsoid of rotation," Mechanics Research Communications, vol. 108, Article ID 103583, 2020.

[26] G. V. Gorr, "Invariant relations and particular solutions of the dynamicsamic equations of a rigid body," Journal of Applied Mathematics and Mechanics, vol. 81, no. 4, pp. 286-294, 2017.

[27] A. A. Galal, T. S. Amer, H. El-Kafly, and W. S. Amer, "The asymptotic solutions of the governing system of a charged symmetric body under the influence of external torques," Results in Physics, vol. 18, Article ID 103160, 2020.

[28] T. S. Amer, A. A. Galal, I. M. Abady, and H. F. Elkafly, "The Dynamicsamical motion of a gyrostat for the irrational frequency case," Applied Mathematical Modelling, vol. 89, pp. 1235-1267, 2021.

[29] A. A. Elmandouh, "On the stability of certain motions of a rigid body-gyrostat in an incompressible ideal fluid," International Journal of Non-linear Mechanics, vol. 120, Article ID 103419, 2020.

[30] H. M. Yehia, "On the motion of a rigid body acted upon by potential and gyroscopic forces: I. the equations of motion and their transformations," Journal of Theoretical and Applied, vol. 5, pp. 747-754, 1986.

[31] M. P. Kharlamov, "Symmetry in systems with gyroscopic forces," Mekh Tverd Tela, vol. 15, pp. 87-93, 1983.

[32] E. Leimanis, The General Problem of Motion of Coupled Rigid Bodies about a Fixed Point, Springer, Berlin, Germany, 1965.

[33] E. T. Whittaker, A Treatise on Analytical Dynamics of Particles and Rigid Bodies, Dover, New York, NY, USA, 1944.

[34] A. V. Borisov and I. S. Mamaev, RigidBodyDynamics-HamiltonianMethods, Integrability Chaos, Institute of Computer Science, Moscow, Russia, in Russian, 2005. 
[35] H. Yehia, "New integrable cases in the dynamics of rigid bodies," Mechanics Research Communications, vol. 13, no. 3, pp. 169-172, 1986.

[36] L. Gavrilov, "Non-integrability of the equations of heavy gyrostat," Compositio Mathematica, vol. 82, p. 27591, 1992.

[37] G. R. Kirchhoff, "Über die bewegung eines körpers in einer flüssigkeit," Journal für die reine und angewandte Mathematik, vol. 1870, no. 71, 2009.

[38] A. Clebsch, "Über die bewegung eines körpers ineiner flüssigkeit," Mathematische Annalen, vol. 238, no. 3, 1871.

[39] H. Lamb, HydroDynamics, Dover Publ., New York, NY, USA, 6th edition, 1945.

[40] H. M. Yehia, "On the motion of a rigid body acted upon by potential and gyroscopic forces.II: a new form of the equations of motion of a multiconnected rigid body in an ideal incompressible fluid," Journal of Mecanical and Theoretical Application, vol. 5, Article ID 755762, 1986.

[41] H. M. Yehia, "Geometric transformations and new integrable problems of rigid body Dynamics," Journal of Physics A: Mathematical and General, vol. 33, no. 23, p. 4393, 2000.

[42] H. M. Yehia, "On the integrability of certain problems in particle and rigid body in an axisymmetric field," Journal of Mecanical and Theoretical Application, vol. 5, pp. 55-71, 1986.

[43] H. M. Yehia, "The master integrable two-dimensional system with a quartic second integral," Journal of Physics A: Mathematical and General, vol. 39, no. 20, pp. 5807-5824, 2006.

[44] H. M. Yehia, "Generalized natural mechanical systems of two degrees of freedom with quadratic integrals," Journal of Physics A: Mathematical and General, vol. 25, Article ID 197221, 1992.

[45] H. M. Yehia, "On certain two dimensional conservative mechanical system with cubic second integral," Journal of Physics A: Mathematical and General, vol. 35, Article ID 94699487, 2002.

[46] A. A. Elmandouh, "New integrable problems in a rigid body Dynamics with cubic integral in velocities," Results in Physics, vol. 8, pp. 559-568, 2018.

[47] H. M. Yehia and A. A. Elmandouh, "Integrable 2D time-irreversible systems with a cubic second integral," Advances in Mathematical Physics, vol. 2016, Article ID 8958747, 10 pages, 2016.

[48] H. M. Yehia and A. A. Elmandouh, "A new conditional integrable case in the dynamics of a rigid body-gyrostat," Mechanics Research Communications, vol. 78, pp. 25-27, 2016.

[49] H. M. Yehia and A. A. Elmandouh, "New conditional integrable cases of motion of a rigid body with kovalevskaya's configuration," Journal of Physics A: Mathematical and Theoretical, vol. 44, Article ID 012001, 2011.

[50] G. D. Birkhoff, Dynamicsamical Systems, American Mathematical Society, New York, NY, USA, 1927.

[51] H. M. Yehia, "Kovalevskaya's integrable case: generalizations and related new results," Regular and Chaotic Dynamics, vol. 8, no. 3, pp. 337-348, 2003.

[52] H. M. Yehia and N. Bedwihy, "Certain generalization of kovalevskaya's case," Mansoura Science Bulletin, vol. 14, pp. 373-386, 1987.

[53] H. M. Yehia, "Two-dimensional conservative mechanical systems with quartic second integral," Regular and Chaotic Dynamics, vol. 11, no. 1, pp. 103-122, 2006.

[54] H. M. Yehia, "New integrable problems in the dynamics of rigid bodies with the kovalevskaya configuration. I - the case of axisymmetric forces," Mechanics Research Communications, vol. 23, no. 5, pp. 423-427, 1996.
[55] D. N. Goriatchev, "New case of integrability of the euler dynamicsamical equations," Varshav University of Izvest, vol. 3, pp. 1-13, 1916.

[56] S. A. Chaplygin, "A new partial solution of the problem of motion of a rigid body in a liquid," Trudy Otdel Fiz Nauk Obsh Lyub Estimation, vol. 11, pp. 7-11, 1903. 